\title{
Niraparib as Maintenance Therapy in Germline ATM-mutated and Somatic BRCA2-mutated Ovarian Cancer with Brain Metastases: A Case Report and Literature Review
}

This article was published in the following Dove Press journal: OncoTargets and Therapy

\author{
Mengyu Tao ${ }^{1,2}$ \\ Jiejun Cheng ${ }^{3}$ \\ Xia $\mathrm{Wu}^{1,2}$ \\ 'Department of Obstetrics \& \\ Gynecology, Renji Hospital, School of \\ Medicine, Shanghai Jiaotong University, \\ Shanghai 200127, People's Republic of \\ China; ${ }^{2}$ Shanghai Key Laboratory of \\ Gynecologic Oncology, Shanghai 200127, \\ People's Republic of China; ${ }^{3}$ Department \\ of Radiology, Renji Hospital, School of \\ Medicine, Shanghai Jiaotong University, \\ Shanghai 200127, People's Republic of \\ China
}

Correspondence: Xia Wu

Department of Gynecology and

Obstetrics, Shanghai Key Laboratory of

Gynecology Oncology, Renji Hospital,

School of Medicine, Shanghai Jiao Tong

University, 160 Pujian Road, Shanghai

200 I27, People's Republic of China

$\mathrm{Tel} / \mathrm{Fax}+86-2 \mathrm{I}-68383062$

Email wuxia1225@aliyun.com

Jiejun Cheng

Department of Radiology, Renji Hospital, School of Medicine, Shanghai Jiao Tong

University, 160 Pujian Road, Shanghai

200 127, People's Republic of China

Email drchij@163.com

\begin{abstract}
Brain metastases from epithelial ovarian cancer are very rare with an incidence of only $1-2.5 \%$. Many therapeutic methods such as surgery, irradiation and chemotherapy do produce survival benefits, but the overall outcome remains unsatisfactory. The BRCA (breast cancer susceptibility gene) mutation status seems to be associated with the development of brain metastases from ovarian cancer and these patients may benefit from treatment with PARP (poly ADP ribose polymerase) inhibitors. Here is a case where a Chinese female patient diagnosed with high-grade serous ovarian cancer with brain metastases was detected to have known germline ATM mutation and somatic BRCA2 mutation. The patient underwent whole brain radiotherapy and systemic chemotherapy, commenced niraparib as maintenance treatment and then presented considerable clinical and radiological response.
\end{abstract}

Keywords: ovarian cancer, brain metastases, $A T M, B R C A$, PARP inhibitors, niraparib

\section{Introduction}

Brain metastasis (BM) is an uncommon and late-stage complication of epithelial ovarian cancer (EOC). The incidence of brain metastasis is between 1 and $2.5 \%$ with an increasing trend in recent reports, probably associated with progressed detection technology and longer survival from ovarian cancer. ${ }^{1}$ Brain metastases often develop two years after primary diagnosis of EOC, accompanied by dismal prognosis with an overall survival (OS) of only 6-12 months, and poor performance status and presence of extracranial lesions have negative impacts on the outcome. $^{2-4}$ Previous studies suggest that multimodality approaches including chemotherapy, radiotherapy, stereotactic radiosurgery (SRS) and resection surgery benefit the survival of brain metastasis, but the long-term prognosis is still unsatisfactory. $^{2}$ The rarity of ovarian cancer brain metastases suffocates the establishment of best treatment consensus.

$B R C A 1 / 2$ gene mutations recognized as genetic predisposition factors for ovarian and breast cancer, were carried by $20 \%$ of high-grade serous ovarian cancer (HGSOC) patients. ${ }^{5}$ Several studies demonstrated an increased risk for brain metastases in patients with EOC associated to $B R C A$ mutations. ${ }^{6-9}$ Despite this, $B R C A 1 / 2$ mutation carriers have been reported to have longer OS and better prognosis as result of higher sensitivity to platinum-based chemotherapy. ${ }^{10,11}$ 
Assessment of BRCA1/2 mutational status also produces a relevant clinical value in selecting patients eligible for surgical therapy in recurrent ovarian cancer. ${ }^{12,13}$

PARP inhibitors (PARPis) induce the formation of doublestrand DNA breaks by "trapping" of PARP1 and blocking the repair pathway of single-stranded DNA breaks. Tumor cells deficient in homologous recombination repair pathway ultimately come to death owing to inability to accurately repair doubled-stand DNA breaks, which is known as synthetic lethality. ${ }^{14,15}$ PARPis have demonstrated clinical benefits in ovarian cancer with homologous recombination deficiency (HRD), particularly effective in BRCA-mutated patients. ${ }^{16,17}$ In December of 2019, the China National Medical Products Administration approved the use of olaparib as frontline maintenance therapy in EOC patients who achieved complete or partial remission by first-line chemotherapy and the application of niraparib as maintenance therapy in patients with platinum-sensitive recurrent ovarian cancer.

Here is a case where a 62-year-old Chinese woman suffered from recurrent ovarian cancer with multiple BM was effectively treated with niraparib as maintenance therapy.

\section{Case Presentation}

A 62-year-old female patient with dizziness and decreased myodynamia for one week was admitted to Renji Hospital of Shanghai Jiaotong University (Shanghai, China) in October 2018. The treatment timeline is shown in Figure 1. For her past history, she underwent primary debulking surgery in November 2017 in another hospital. The postoperative specimen revealed high-grade serous epithelial ovarian cancer involving bilateral ovaries, fallopian tubes and omentum. Intraoperative exploration suggested the diagnosis of HGSOC with FIGO stage IIIC due to extensive peritoneal dissemination especially omental implants measuring $20 * 8 * 2 \mathrm{~cm}$. The patient then received intravenous chemotherapy with taxol $\left(175 \mathrm{mg} / \mathrm{m}^{2}, \mathrm{~d} 1\right)$ and carboplatin (area under the curve; $\mathrm{AUC}=5, \mathrm{~d} 1$ ). After six cycles of the same chemotherapy regime, no residual disease was found on CT scan and the level of CA125 was normal. The patient kept routine follow-up and remained well for seven months.

Dizziness occurred in October 2018, accompanied by decreased myodynamia of the left upper and lower limbs. On physical examination, myodynamia of her right upper limb was grade V, left upper limb was grade II, and both lower limbs were grade IV. In addition, there were no other positive neurological signs and the laboratory test findings of tumor markers were all within the normal limits. Her serum level of CA125 was $30.41 \mathrm{U} / \mathrm{mL}$. CT head scan demonstrated multiple lesions within bilateral frontal lobe, parietal lobe and right cerebellum as well as MRI-detected multiple BMs at the same locations (Figure 2). Although we did not have a biopsy of intracranial lesions to confirm metastasis over primary carcinoma, the performance of 18F-FDG PET/ $\mathrm{CT}$ consistent with metastatic lesions of lesser sac and lymph nodes was a reliable basis for diagnosis (Figure 3). After obtaining informed consent from the patient, we performed next generation sequence of her primary tumor tissue from the primary debulking surgery and paired peripheral blood. The next generation sequencing panel included 508 cancerrelated genes which weres run on a BGISEQ-2000 platform. This test detected five clinically significant variations included four somatic mutations and one germline mutation (Table 1). The four somatic mutations included BRCA2 nonsense mutation (c.7171G>T, p.E2391*), NF1 nonsense mutation (c.3367G $>$ T, p.E1123*), NF2 nonsense mutation (c.361G $>\mathrm{T}, \mathrm{p} . \mathrm{E} 121^{*}$ ) and CREBBP missense mutation (c. $4054 \mathrm{C}>\mathrm{G}$, p.P1352A). The germline mutation was $A T M$ nonsense mutation (c.3609del, p.Y1203*), which is a pathogenic mutation predicted to cause truncation of the ATM protein. ATM as member of the PI3K related kinase family plays a key role in homologous recombination repair and the loss function mutation of ATM may lead to HRD.

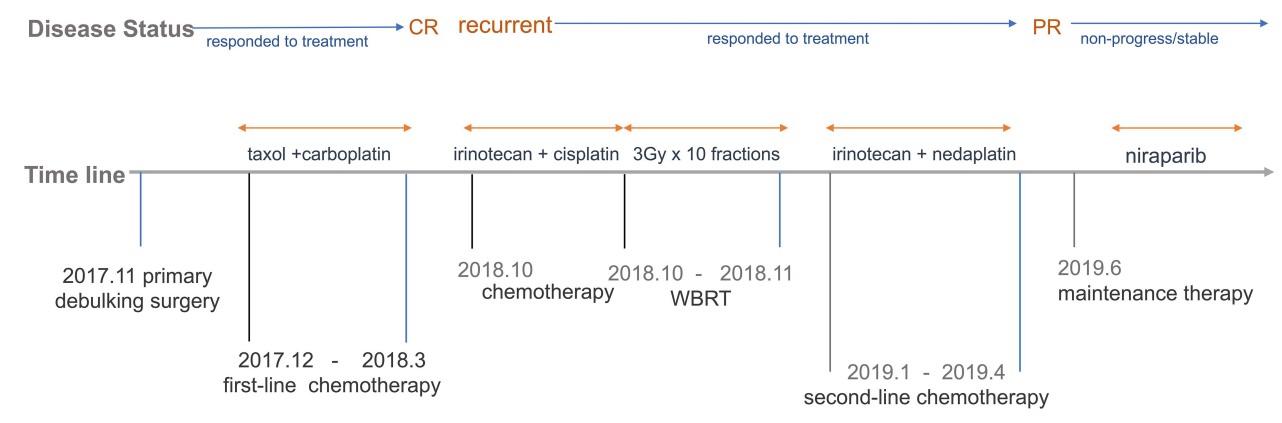

Figure I Timeline of different treatments and disease status. 

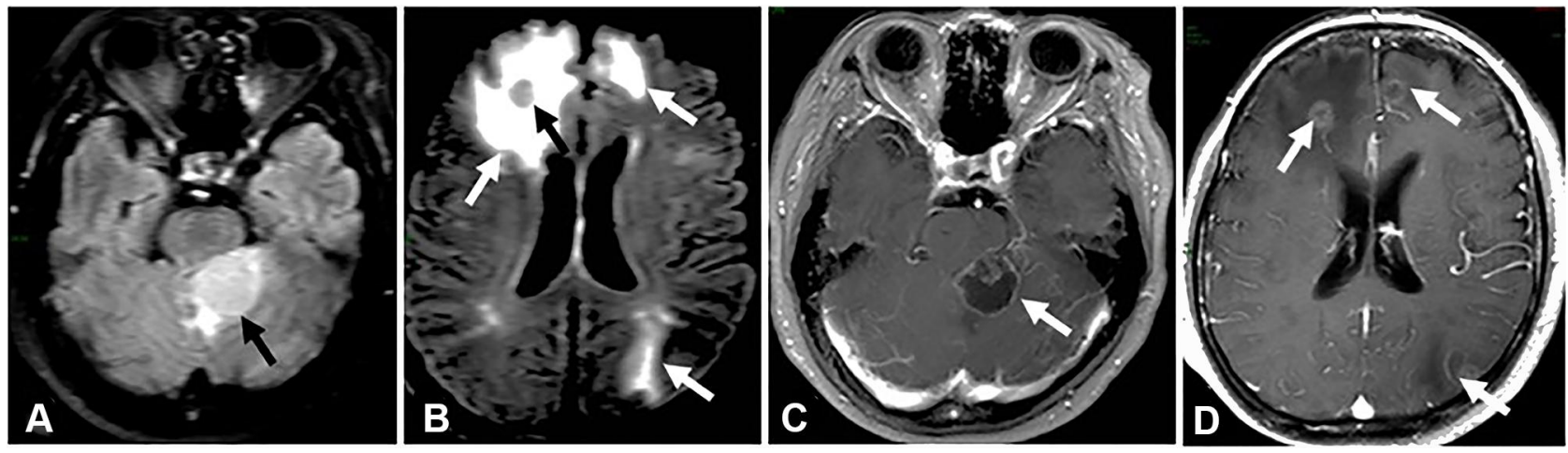

Figure 2 MRI baseline of brain metastases. (A) Axial T2-weighted fluid-attenuated inversion recovery (FLAIR) shows circular high signal (black arrow) of the left anterior border of cerebellum. (B) Axial T2-weighted FLAIR shows patchy edema of bilateral frontal and left occipital lobes (white arrows) and right frontal lobes signals such as nodules (black arrow). (C) Axial gadolinium-enhanced TI-weighted MRI shows cerebellar left front ring mass with peripheral rim enhancement surrounding a low-signalintensity area of central necrosis (white arrow). (D) Axial gadolinium-enhanced TI-weighted MRI shows bilateral frontal lobe and left occipital small ring enhancement (white arrows).

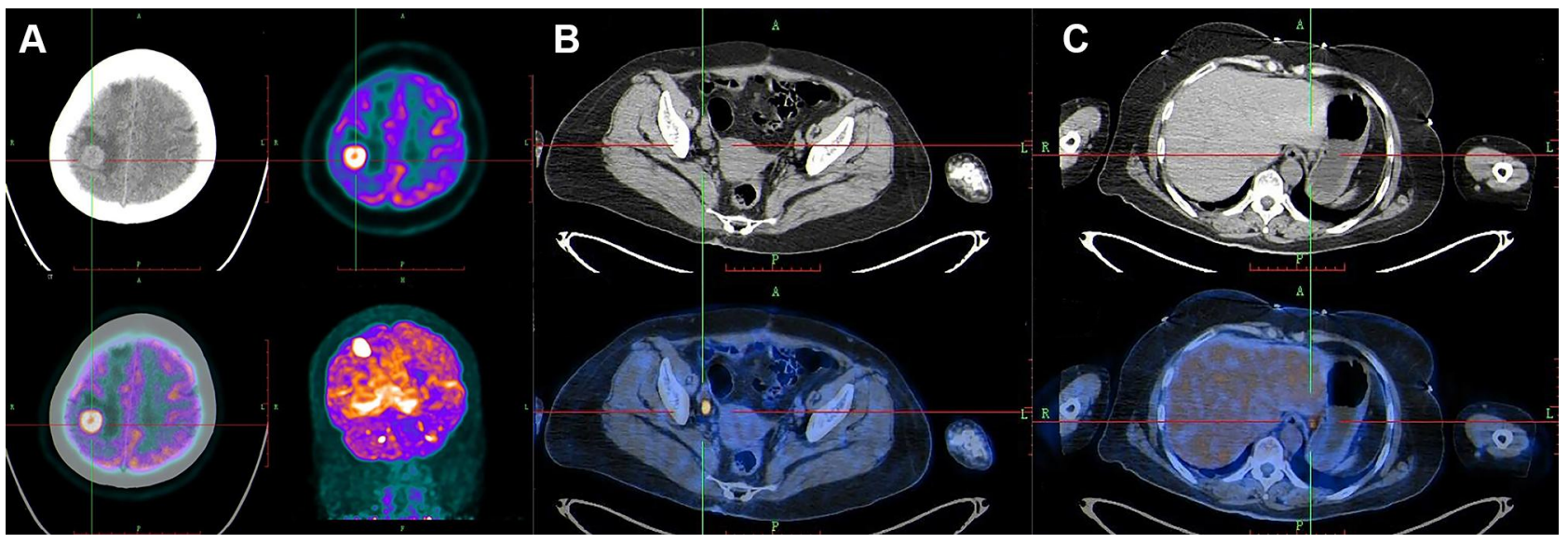

Figure 3 PET-CT showed recurrence and metastases of ovarian cancer. Increased I8F-FDG uptake was shown in brain (A), right para-aortic lymph nodes (B), and lesser sac (C).

Due to the coexistence of both abdominal cavity metastasis and intracranial lesions, the patient was treated with one cycle of intravenous chemotherapy with irinotecan $\left(140 \mathrm{mg} / \mathrm{m}^{2}, \mathrm{~d} 1\right)$ and cisplatin $\left(75 \mathrm{mg} / \mathrm{m}^{2}, \mathrm{~d} 1\right)$. Then she received whole-brain irradiation (WBRT) and the target was given $30 \mathrm{~Gy}$ in total in 10 fractions. By the end of radiotherapy, she developed a series of side effects like fatigue, somnolence, anorexia, and dermatitis, which then improved by symptomatic support treatment. Afterwards the patient received systematic intravenous chemotherapy with irinotecan $\left(140 \mathrm{mg} / \mathrm{m}^{2}, \mathrm{~d} 1\right)$ and nedaplatin $\left(80 \mathrm{mg} / \mathrm{m}^{2}, \mathrm{~d} 1\right)$ for five cycles in total. A remission of brain metastases was then detected by head MRI after WBRT and chemotherapy (Figure 4), and her CA125 reduced from $46.8 \mathrm{IU} / \mathrm{mL}$ to $23.7 \mathrm{IU} / \mathrm{mL}$.

Table I Gene Mutation

\begin{tabular}{|c|c|c|c|c|c|}
\hline \multirow[t]{2}{*}{ Characteristics } & \multicolumn{4}{|c|}{ Somatic Gene } & \multirow{2}{*}{$\begin{array}{l}\text { Germline Gene } \\
\text { ATM }\end{array}$} \\
\hline & BRCA2 & NFI & NF2 & CREBBP & \\
\hline Nucleotide change & c.7|7| IG >T & $c .3367 G>T$ & c. $36 \mid \mathrm{G}>\mathrm{T}$ & c. $4054 \mathrm{G}>\mathrm{T}$ & c. $3609 \mathrm{del}$ \\
\hline Amino acid change & p.E239/ ${ }^{\mathrm{a}}$ & p. $1123^{a}$ & p. $121^{\mathrm{a}}$ & p.PI352A & p.YI $203^{a}$ \\
\hline Gene region & EXI4 & EX26 & EX5 & EX24 & $\mathrm{EX} 25$ \\
\hline Frequency & $53.01 \%$ & $35.79 \%$ & $15.21 \%$ & $30.98 \%$ & - \\
\hline Transcript & NM_000059.3 & NM_000267.3 & NM_181830.2 & NM_004380.2 & NM_00005.3 \\
\hline
\end{tabular}

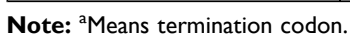



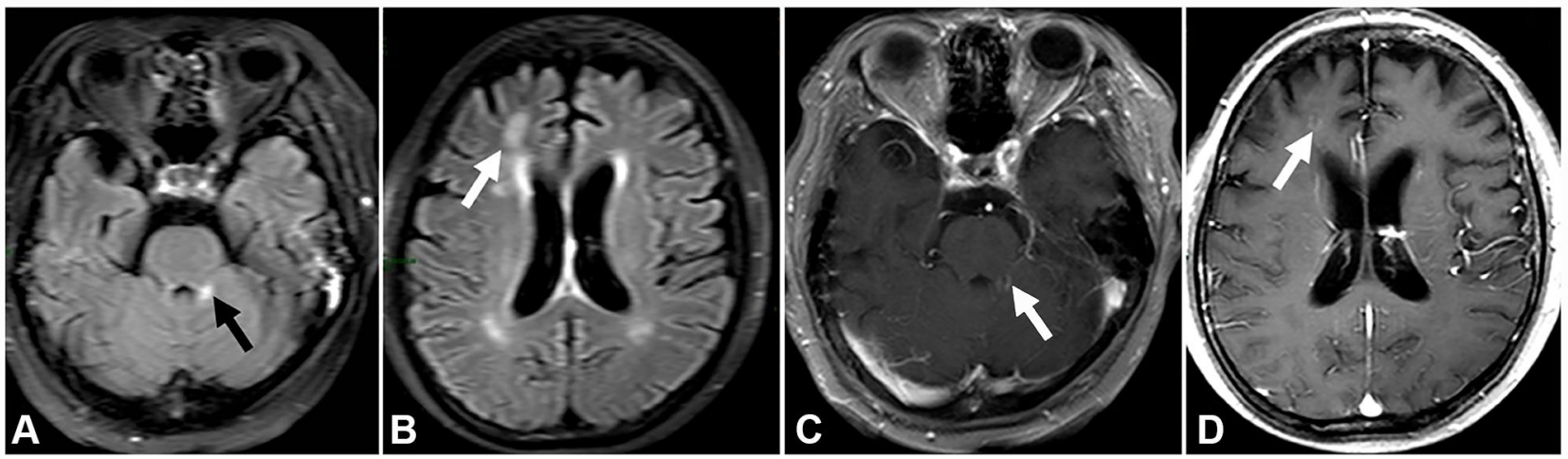

Figure 4 Head MRI after whole-brain irradiation and chemotherapy. (A) Axial T2-weighted FLAIR shows small spot high signal (black arrow) of the left anterior border of cerebellum. (B) Axial T2-weighted FLAIR shows patchy edema of right frontal lobes (white arrow). (C) Axial gadolinium-enhanced TI-weighted image shows cerebellar left front small spot enhancement (white arrow). (D) Axial gadolinium-enhanced TI-weighted image shows right frontal lobe small spot enhancement (white arrow).

The patient commenced niraparib capsules $200 \mathrm{mg}$ orally once daily as maintenance therapy since June 2019 and the result of routine blood test ten days later was normal. The treatment was suspended for two weeks because she developed bone marrow suppression (neutropenia grade 2, thrombocytopenia grade 3 based on the National Cancer Institute Common Toxicity Criteria for Adverse Events version 4.0). When recovered from the bone marrow suppression, the patient continued niraparib treatment again, with $100 \mathrm{mg}$ once daily for a month. Since August 2019, the dosage has been increased to $200 \mathrm{mg}$ once daily and the patient is currently on this treatment regimen. After nine months of niraparib administration, BM almost disappeared on the head MRI (Figure 5). In addition, abdominal contrast-enhance CT scan also revealed no evidence of disease progression. In the past 15 months after the initiation of niraparib treatment, the patient has tolerated well without the severe side effects such as nausea, asthenia, and fatigue.

\section{Discussion}

Brain metastases are considered to be rare and advanced manifestations of ovarian cancer. Since whole body radiological examinations such as PET/CT have been included in routine assessment increasingly, there are likely to be more reports of asymptomatic brain metastases. ${ }^{2}$ There are several indices to assess the prognosis of patients with brain metastasis from ovarian cancer, generally taking account for patients' age, number, and sites of lesions, existence of extracranial metastases, performance status, and primary disease status. ${ }^{18}$ So far, there is no agreed consensus on optimal treatment of ovarian cancer with BM. Resection surgery and gamma-knife radiosurgery have been shown effective for solitary metastasis, but they have limited feasibility in patients with multiple lesions or poor performance status. ${ }^{19,20}$ Furthermore, surgical approach combined with radiotherapy such as WBRT or SRS, offers better advantages than either of them individually. ${ }^{21}$ Platinum-based chemotherapy as an excellent systematic control approach, has limited efficiency in preventing central nervous system tumor spread though, owing to insufficient penetration to the blood-brain barrier (BBB). However, studies have shown that WBRT can increase the permeability of the BBB to chemotherapeutic drugs. $^{22,23}$ Thus, WBRT combined with chemotherapy has been the most common treatment for patients with multiple BM. ${ }^{1,4}$ In this case, we chose irinotecan with platinum as the chemotherapy regimen for this platinum-sensitive relapsed ovarian cancer patient with BM. Irinotecan, a semi-synthetic water-soluble cytotoxic quinoline alkaloid camptothecin analog, is activated by hydrolysis to SN-38, an inhibitor of topoisomerase I. Irinotecan has demonstrated activity with the response rate of $52 \%$ as a combination therapy in platinum-sensitive relapse ovarian cancer. ${ }^{24,25}$ Furthermore, the ability of irinotecan to cross the BBB has been demonstrated in primary brain tumors. ${ }^{26}$ Preliminary research shows that irinotecan and SN-38 are transported across the BBB with brain/plasma ratios of 2.1 and 2.3 respectively. ${ }^{27}$

A retrospective analysis on clinical data from the Flatiron Health database, which is a longitudinal, demographically, and geographically diverse database derived from electronic health records data in the USA, was conducted by Ratner et al to estimate the association between $B R C A$ mutations and the risk of brain metastasis in ovarian cancer patients. ${ }^{8}$ Among 4515 patients diagnosed with ovarian 


\section{A After 3 months}

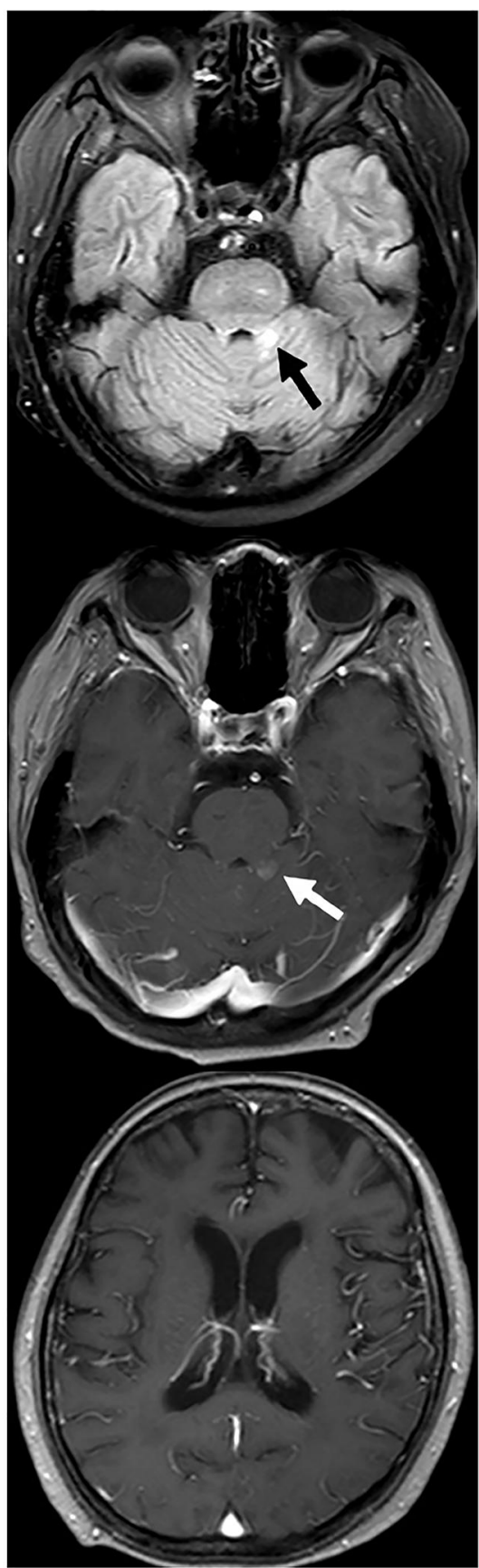

B After 6 months

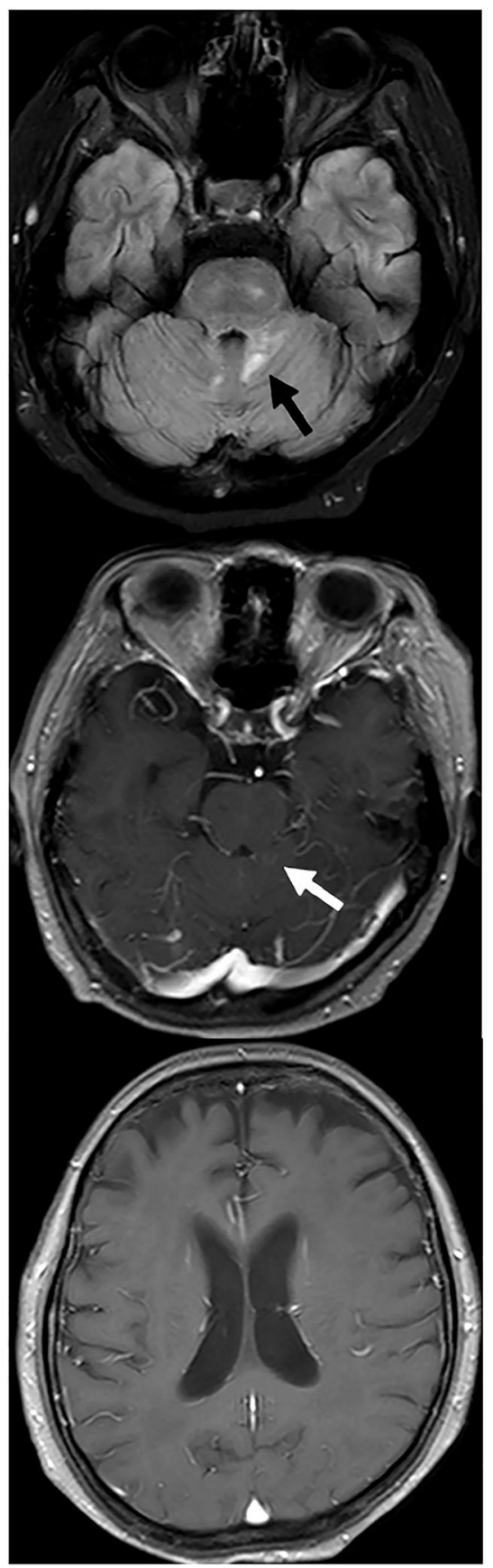

\section{After 9 months}

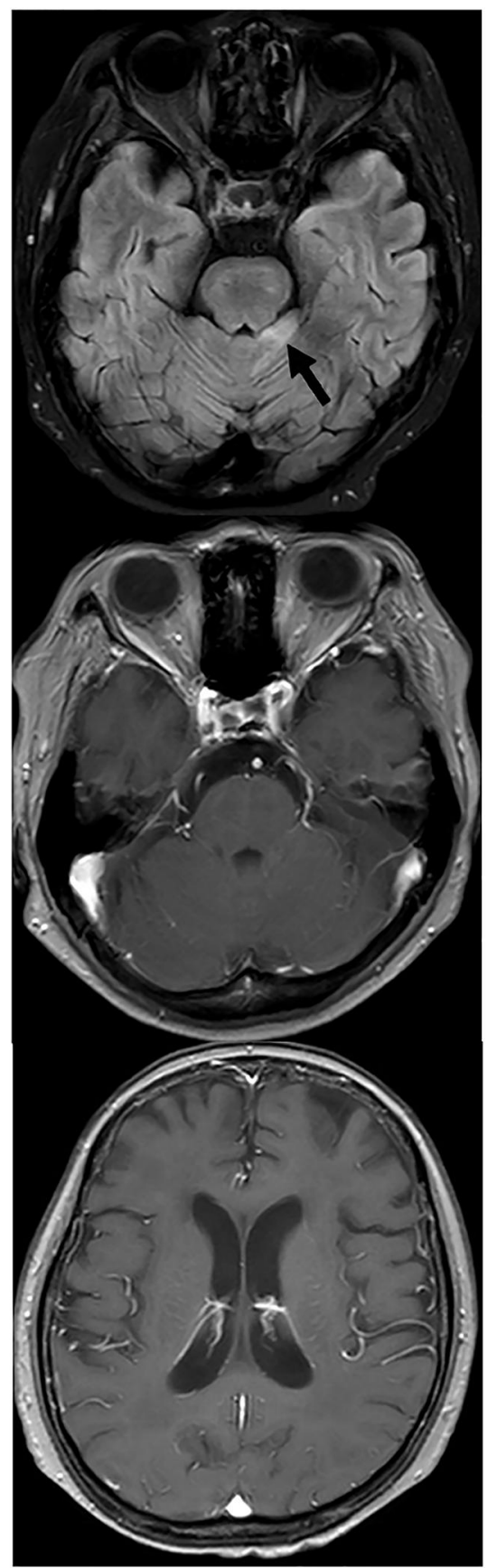

Figure 5 Head MRI after niraparib maintenance therapy. (A) after three months of niraparib. (B) After six months of niraparib. (C) After nine months of niraparib. Axial T2weighted FLAIR shows reduction of small spot high signal of the left anterior border of cerebellum (black arrow), Axial gadolinium-enhanced TI-weighted MRI shows vanish of cerebellar left front tiny dot enhancement (white arrow) and of the right frontal lobe tiny dot enhancement.

cancer, 2206 (49\%) underwent $B R C A$ testing. Patients were defined as having a $B R C A$ mutation if they carried either a $B R C A 1 / 2$ mutation, genetic variant favor polymorphism, genetic variant of unknown significance, or $B R C A$ mutation not otherwise specified. Of patients with confirmed $B R C A$ status (germline or somatic mutation), brain metastases were observed in $3 \%$ of patients with $B R C A$ mutations compared with $0.6 \%$ of those with $B R C A w t$. The data demonstrated that patients with a $B R C A$ mutation had a significantly higher risk for brain metastases than those without. ${ }^{8}$ The mutation profile of ovarian cancer brain metastases was assessed by Balendran et al through next-generation 
sequencing. ${ }^{9}$ In total, seven out of eight samples carried either a $B R C A 1$ or a $B R C A 2$ pathogenic alteration, with fiveout of eight patients showing a BRCA1 mutation and two out of eight a $B R C A 2$ mutation. $B R C A$-mutations were confirmed by an independent analysis in four patients, and two cases were further proved to be of somatic origin by testing matched normal tissue. Due to these results, the development of brain metastases is probably relevant to both germline and somatic $B R C A$ mutations. Furthermore, all eight $\mathrm{BM}$ samples revealed mutations in at least one DNA repair gene and most commonly mutated genes were BRCA1/2, TP53, and ATM. ${ }^{9}$ However, whether the BRCA and DNA repair malfunction is functionally related to ovarian cancer cells metastasizing to the brain remains unclear, hence, the mechanism confirming the correlation needs to be further explored. The knowledge of mutation status of DNA homologous recombination associated protein in ovarian cancer patients with BM is of vital importance, because some patients may benefit more from targeted therapeutic of PARP inhibitors.

In animal models, niraparib penetrates the $\mathrm{BBB}$ and betrays good sustainability in the brain, whereas olaparib was eliminated from the brain quickly. Compatible with its favorable tumor and brain distribution, niraparib accomplishes more powerful growth inhibition of tumor than olaparib in an intracranial tumor model at greatest tolerable doses. $^{28}$ Although in preclinical models it was shown that niraparib can cross the BBB and show efficacy, the role of PARPis as maintenance therapy in ovarian cancer with intracranial metastases is not known from clinical trials. Until now, PARPis as maintenance therapy have been confirmed beneficial in terms of PFS in platinum sensitive recurrent ovarian cancer, especially for those with BRCA1/ 2 mutations, in one Phase II (Study 19) and three Phase III clinical trials (SOLO2, NOVA, and ARIEL3). ${ }^{29-32}$ However, in Study 19, SOLO-2 and ARIEL3 trials, the enrollment criteria have excluded patients with symptomatic or untreated central nervous system metastases. In the NOVA trial, it is unknown whether there are patients with brain metastases enrolled. Under these circumstances, limited case reports about the use of PARP inhibitors in ovarian cancer patients with brain metastasis provide valuable reference to clinical practice. After searching carefully through PubMed, we found that only five cases in total (including three ovarian cancer patients, one endometrial cancer patient and one primary peritoneal cancer patient) of successful treatment of brain metastases with PARP inhibitors have been reported (Table 2).

To the best of our knowledge, this is the first report that explains the efficacy of niraparib in a Chinese patient with multiple BMs from germline ATM and somatic BRCA2 mutated HGSOC. The patient achieved partial remission after receiving WBRT and systemic chemotherapy, and the lesions of brain metastases continued to decease after initiation of niraparib therapy. The cerebellar left front tiny dot enhancement and the right frontal lobe tiny dot enhancement totally disappeared on the head MRI after nine months of niraparib treatment (Figure 5C). The reported OS of EOC patients with multiple BM is only 6-12 months, in our case, however, the patient has maintained a very good functional status without disease progression for nearly two years (up to now) after the onset of BMs. What is noteworthy is that the patient has both germline $A T M$ and somatic BRCA2 mutations. ATM, a member of the $\mathrm{PI} 3 \mathrm{~K}$ related kinase family, is involved in mediating the cellular response to doubledstand DNA breaks and replication stress. ATM operates as a key enzyme in homologous recombination repair, and the loss function mutation of ATM may lead to HRD. ${ }^{33-35}$ As we known, due to the DNA repair defect, $B R C A 1 / 2$ deficient or HRD-positive tumor cells are more sensitive to PARPis through the mechanism of synthetic lethality. In the NOVA trial, niraparib treatment resulted in significantly longer PFS than placebo in both the $\mathrm{g} B R C A$ subset

Table 2 Five Cases of Successful Treatment for Brain Metastases with PARP Inhibitors

\begin{tabular}{|c|c|c|c|c|c|c|c|}
\hline Authors \& Year & Tumor Type & $\begin{array}{l}\text { FIGO } \\
\text { Stage }\end{array}$ & $\begin{array}{l}\text { Number } \\
\text { of BM }\end{array}$ & $\begin{array}{l}\text { Extracranial } \\
\text { Lesions }\end{array}$ & $\begin{array}{l}\text { Gene } \\
\text { Mutation }\end{array}$ & PARPis & PFS \\
\hline Forster et al, $201 \mathrm{I}^{36}$ & $\begin{array}{l}\text { Endometrioid endometrial } \\
\text { carcinoma }\end{array}$ & IVB & $>2$ & $\begin{array}{l}\text { Lungs, liver, } \\
\text { peritoneum }\end{array}$ & PTEN & Olaparib & $>10$ months \\
\hline Bangham et al, $2016 .^{37}$ & Ovarian cancer & IVB & 1 & None & $B R C A 2$ & Olaparib & 12 months \\
\hline Sakamoto et al, $2019 .{ }^{38}$ & Primary peritoneal cancer & IIIC & $>2$ & None & $B R C A I$ & Olaparib & 22 months \\
\hline Gray et al, $2019^{39}$ & Ovarian cancer & IIIC & $>2$ & None & $B R C A I$ & Niraparib & 17 months \\
\hline Favier et al, $2020 .^{40}$ & Ovarian cancer & IIIC & - & Peritoneum & $B R C A 2$ & Olaparib & 14 months \\
\hline
\end{tabular}


(median PFS, 21.0 vs 5.5 months) and the HRD-positive subset including somatic BRCA mutation (median PFS, 12.9 vs 3.8 months). In our case, the great benefit from niraparib the patient has gained probably lies in the functional loss of both $A T M$ and BRCA2, which contributes to the synthetic lethality induced by niraparib.

\section{Conclusion}

Because of the rarity of BM occurrence in EOC disease, there is no consensus regarding the best management of EOC patients with BMs, and the efficacy of PARPis as monotherapy maintenance in platinum-sensitive recurrent EOC patients with BMs is almost completely unknown. In our case, the patient underwent whole brain radiotherapy and systemic chemotherapy, commenced niraparib as maintenance therapy and then presented very durable, considerable clinical and radiological response. This case brings about very important reference value for handling similar patients in clinical practice. Future clinical trials could focus on a larger group of patients with $\mathrm{BM}$ related to $B R C A$ mutated or HRD HGSOC to assess the use of PARPis in this setting.

\section{Abbreviations}

PARP, poly adenosine diphosphate ribose polymerase; BM, brain metastasis; EOC, epithelial ovarian cancer; OS, overall survival; PFS, progression-free survival; $\mathrm{CR}$, complete response; PR, partial response; SRS, stereotactic radiosurgery; HGSOC, high-grade serous ovarian cancer; HRD, homologous recombination deficiency; WRBT, whole-brain radiotherapy; $C R E B B P$, cyclic adenosine monophosphate response element binding protein; $N F$, neurofibromatosis type; PI3K, phosphatidylinositol 3-kinase; BBB, blood-brain barrier.

\section{Ethics and Consent Statement}

The patient agreed and submitted a written informed consent to allow publication of the details of the case. An institutional approval was not required for a case report.

\section{Disclosure}

The authors report no conflicts of interest in this work.

\section{References}

1. Pietzner K, Oskay-Oezcelik G, El Khalfaoui K, Boehmer D, Lichtenegger W, Sehouli J. Brain metastases from epithelial ovarian cancer: overview and optimal management. Anticancer Res. 2009;29 (7):2793-2798.
2. Marchetti C, Ferrandina G, Cormio G, et al. Brain metastases in patients with EOC: clinico-pathological and prognostic factors. A multicentric retrospective analysis from the MITO group (MITO 19). Gynecol Oncol. 2016;143(3):532-538. doi:10.1016/j.ygyno.2016.09.025

3. Cohen ZR, Suki D, Weinberg JS, et al. Brain metastases in patients with ovarian carcinoma: prognostic factors and outcome. J Neurooncol. 2004;66(3):313-325. doi:10.1023/B:NEON.0000014516.04943.38

4. Pakneshan S, Safarpour D, Tavassoli F, Jabbari B. Brain metastasis from ovarian cancer: a systematic review. J Neurooncol. 2014;119 (1):1-6. doi:10.1007/s11060-014-1447-9

5. Kuchenbaecker KB, Hopper JL, Barnes DR, et al. Risks of breast, ovarian, and contralateral breast cancer for BRCA1 and BRCA2 mutation carriers. JAMA. 2017;317(23):2402-2416. doi:10.1001/ jama.2017.7112

6. Stasenko M, Cybulska P, Feit N, et al. Brain metastasis in epithelial ovarian cancer by BRCA1/2 mutation status. Gynecol Oncol. 2019;154(1):144-149. doi:10.1016/j.ygyno.2019.05.004

7. Sekine M, Yoshihara K, Komata D, Haino K, Nishino K, Tanaka K. Increased incidence of brain metastases in BRCA1-related ovarian cancers. J Obstet Gynaecol Res. 2013;39(1):292-296. doi:10.1111/ j.1447-0756.2012.01961.x

8. Ratner E, Bala M, Louie-Gao M, Aydin E, Hazard S, Brastianos PK Increased risk of brain metastases in ovarian cancer patients with BRCA mutations. Gynecol Oncol. 2019;153(3):568-573. doi:10. 1016/j.ygyno.2019.03.004

9. Balendran S, Liebmann-Reindl S, Berghoff AS, et al. Next-generation sequencing-based genomic profiling of brain metastases of primary ovarian cancer identifies high number of BRCA-mutations. J Neurooncol. 2017;133(3):469-476. doi:10.1007/s11060-017-2459-Z

10. Pennington KP, Walsh T, Harrell MI, et al. Germline and somatic mutations in homologous recombination genes predict platinum response and survival in ovarian, fallopian tube, and peritoneal carcinomas. Clin Cancer Res. 2014;20(3):764-775. doi:10.1158/ 1078-0432.CCR-13-2287

11. Norquist BM, Brady MF, Harrell MI, et al. Mutations in homologous recombination genes and outcomes in ovarian carcinoma patients in GOG 218: an NRG oncology/gynecologic oncology group study. Clin Cancer Res. 2018;24(4):777-783. doi:10.1158/1078-0432.CCR-17-1327

12. Gallotta V, Conte C, D'Indinosante M, et al. Prognostic factors value of germline and somatic brca in patients undergoing surgery for recurrent ovarian cancer with liver metastases. Eur J Surg Oncol. 2019;45(11):2096-2102. doi:10.1016/j.ejso.2019.06.023

13. Gallotta V, Bruno M, Conte C, et al. Salvage lymphadenectomy in recurrent ovarian cancer patients: analysis of clinical outcome and BRCA1/2 gene mutational status. Eur J Surg Oncol. 2020;46 (7):1327-1333. doi:10.1016/j.ejso.2020.01.035

14. Ledermann JA, Drew Y, Kristeleit RS. Homologous recombination deficiency and ovarian cancer. Eur $J$ Cancer. 2016;60:49-58. doi:10.1016/j.ejca.2016.03.005

15. Murai J, Huang SY, Das BB, et al. Trapping of PARP1 and PARP2 by clinical PARP inhibitors. Cancer Res. 2012;72(21):5588-5599. doi:10.1158/0008-5472.CAN-12-2753

16. Moore K, Colombo N, Scambia G, et al. Maintenance olaparib in patients with newly diagnosed advanced ovarian cancer. $N$ Engl $J$ Med. 2018;379(26):2495-2505. doi:10.1056/NEJMoa1810858

17. González-Martín A, Pothuri B, Vergote I, et al. Niraparib in patients with newly diagnosed advanced ovarian cancer. $N$ Engl $J$ Med. 2019;381(25):2391-2402. doi:10.1056/NEJMoa1910962

18. Kim TJ, Song S, Kim CK, et al. Prognostic factors associated with brain metastases from epithelial ovarian carcinoma. Int $J$ Gynecol Cancer. 2007;17(6):1252-1257. doi:10.1111/j.1525-1438.2007.00 941.x

19. Keskin S, Kucucuk S, Ak N, et al. Survival impact of optimal surgical cytoreduction in recurrent epithelial ovarian cancer with brain metastasis. Oncol Res Treat. 2019;42(3):101-106. doi:10. $1159 / 000494334$ 
20. Nikolaoul M, Stamenkovic S, Stergiou C, Skarleas C, Torrens M. Management of brain metastasis in a patient with advanced epithelial ovarian carcinoma by gamma-knife radiosurgery. Srp Arh Celok Lek. 2015;143(3-4):205-209. doi:10.2298/SARH1504205N

21. Kwon JW, Yoon JH, Lim MC, et al. Treatment results and prognostic factors of brain metastases from ovarian cancer: a single institutional experience of 56 patients. Int $J$ Gynecol Cancer. 2018;28 (8):1631-1638. doi:10.1097/IGC.0000000000001341

22. Sprowls SA, Arsiwala TA, Bumgarner JR, et al. Improving CNS delivery to brain metastases by blood-tumor barrier disruption. Trends Cancer. 2019;5(8):495-505. doi:10.1016/j.trecan.2019.06.003

23. Teng F, Tsien CI, Lawrence TS, Cao Y. Blood-tumor barrier opening changes in brain metastases from pre to one-month post radiation therapy. Radiother Oncol. 2017;125(1):89-93. doi:10.1016/j.radonc.2017.08.006

24. Matsumoto K, Katsumata N, Yamanaka Y, et al. The safety and efficacy of the weekly dosing of irinotecan for platinum- and taxanes-resistant epithelial ovarian cancer. Gynecol Oncol. 2006;100 (2):412-416. doi:10.1016/j.ygyno.2005.10.013

25. Tsubamoto H, Kawaguchi R, Ito K, et al. Phase II study of carboplatin and weekly irinotecan combination chemotherapy in recurrent ovarian cancer: a Kansai clinical oncology group study (KCOG0330). Anticancer Res. 2013;33(3):1073-1079.

26. Vredenburgh JJ, Desjardins A, Herndon JE 2nd, et al. Bevacizumab plus irinotecan in recurrent glioblastoma multiforme. J Clin Oncol. 2007;25(30):4722-4729. doi:10.1200/JCO.2007.12.2440

27. Goldwirt L, Beccaria K, Carpentier A, Farinotti R, Fernandez C. Irinotecan and temozolomide brain distribution: a focus on ABCB1. Cancer Chemother Pharmacol. 2014;74(1):185-193. doi:10.1007/ s00280-014-2490-0

28. Sun K, Mikule K, Wang Z, et al. A comparative pharmacokinetic study of PARP inhibitors demonstrates favorable properties for niraparib efficacy in preclinical tumor models. Oncotarget. 2018;9 (98):37080-37096. doi:10.18632/oncotarget.26354

29. Coleman RL, Oza AM, Lorusso D, et al. Rucaparib maintenance treatment for recurrent ovarian carcinoma after response to platinum therapy (ARIEL3): a randomised, double-blind, placebo-controlled, Phase 3 trial. Lancet. 2017;390(10106):1949-1961. doi:10.1016/ S0140-6736(17)32440-6

30. Ledermann J, Harter P, Gourley C, et al. Olaparib maintenance therapy in patients with platinum-sensitive relapsed serous ovarian cancer: a preplanned retrospective analysis of outcomes by BRCA status in a randomised Phase 2 trial. Lancet Oncol. 2014;15 (8):852-861. doi:10.1016/S1470-2045(14)70228-1
31. Mirza MR, Monk BJ, Herrstedt J, et al. Niraparib maintenance therapy in platinum-sensitive, recurrent ovarian cancer. $N$ Engl $J$ Med. 2016;375(22):2154-2164. doi:10.1056/NEJMoa1611310

32. Pujade-Lauraine E, Ledermann JA, Selle F, et al. Olaparib tablets as maintenance therapy in patients with platinum-sensitive, relapsed ovarian cancer and a BRCA1/2 mutation (SOLO2/ENGOT-Ov21): a double-blind, randomised, placebo-controlled, phase 3 trial. Lancet Oncol. 2017;18(9):1274-1284. doi:10.1016/S1470-2045(17)30469-2

33. Morrison C, Sonoda E, Takao N, Shinohara A, Yamamoto K, Takeda S. The controlling role of ATM in homologous recombinational repair of DNA damage. EMBO J. 2000;19(3):463-471. doi:10.1093/emboj/19.3.463

34. Beucher A, Birraux J, Tchouandong L, et al. ATM and Artemis promote homologous recombination of radiation-induced DNA double-strand breaks in G2. EMBO J. 2009;28(21):3413-3427. doi:10.1038/emboj.2009.276

35. McCabe N, Turner NC, Lord CJ, et al. Deficiency in the repair of DNA damage by homologous recombination and sensitivity to poly (ADP-ribose) polymerase inhibition. Cancer Res. 2006;66 (16):8109-8115. doi:10.1158/0008-5472.CAN-06-0140

36. Forster MD, Dedes KJ, Sandhu S, et al. Treatment with olaparib in a patient with PTEN-deficient endometrioid endometrial cancer. Nat Rev Clin Oncol. 2011;8(5):302-306. doi:10.1038/nrclinonc. 2011.42

37. Bangham M, Goldstein R, Walton H, Ledermann JA. Olaparib treatment for BRCA-mutant ovarian cancer with leptomeningeal disease. Gynecol Oncol Rep. 2016;18:22-24. doi:10.1016/j.gore. 2016.10.004

38. Sakamoto I, Hirotsu Y, Nakagomi H, Ikegami A, Teramoto K, Omata M. Durable response by olaparib for a Japanese patient with primary peritoneal cancer with multiple brain metastases: a case report. J Obstet Gynaecol Res. 2019;45(3):743-747. doi:10.1111/ jog. 13851

39. Gray S, Khor XY, Yiannakis D. Niraparib as maintenance therapy in a patient with ovarian cancer and brain metastases. BMJ Case Rep. 2019;12(8):e230738. doi:10.1136/bcr-2019-230738

40. Favier L, Truc G, Boidot R, Bengrine-Lefevre L. Long-term response to Olaparib in Carcinomatous meningitis of a BRCA2 mutated ovarian cancer: A case report. Mol Clin Oncol.2020;13(1):73-75.

\section{Publish your work in this journal}

OncoTargets and Therapy is an international, peer-reviewed, open access journal focusing on the pathological basis of all cancers, potential targets for therapy and treatment protocols employed to improve the management of cancer patients. The journal also focuses on the impact of management programs and new therapeutic agents and protocols on patient perspectives such as quality of life, adherence and satisfaction. The manuscript management system is completely online and includes a very quick and fair peer-review system, which is all easy to use. Visit http://www.dovepress.com/ testimonials.php to read real quotes from published authors. 